\title{
Thoracoscopic Upper Thoracic Sympathectomy for Upper Limb Hyperhidrosis
}

\author{
M.M. Farghaly ${ }^{a}$ M.S. Al-Manee ${ }^{a}$ A.K. Ayed ${ }^{b}$ M.A. Khoursheed ${ }^{b}$ \\ Department of Surgery, ${ }^{a} \mathrm{Al}-$ Adan Hospital and ${ }^{\mathrm{b}}$ Faculty of Medicine, Kuwait
}

\section{Key Words}

Axilla . Compensatory hyperhidrosis .

Ganglia $\cdot$ Hand $\cdot$ Pleural $\cdot$ Pneumothorax ·

Adhesions · Thoracoscopic sympathectomy

\begin{abstract}
Objectives: To present a 30-month experience in thoracoscopic upper thoracic sympathectomy (TUTS) in Kuwait in 20 patients with upper limb hyperhidrosis (ULH). Methods: We performed 40 TUTSs in 20 patients with bilateral ULH. The T2-4 or T2-5 sympathetic ganglia were resected in 36 cases. Only the T2-3 ganglia were resected in 4 cases due to difficult localization of the 4th ganglion. Both sides were performed simultaneously. Chest tube drainage was performed at the end of each procedure. Results: The male:female ratio was 9:1, ranging in age from 13 to 43 years (mean age 26.95 years).
\end{abstract}

Two patients had bilateral dense pleural adhesions, but the procedure was successful. Most patients were discharged on the 2 nd postoperative day. Follow-up at 5-30 months (mean 16.3 months) was excellent both for immediate and permanent relief of ULH. Eleven of the 15 patients with pedal hyperhidrosis also had dry feet. Two patients developed transient Horner's eye syndrome. One patient had persistent right pneumothorax that required chest tube drainage for 7 days. Four patients developed compensatory hyperhidrosis of the trunk and upper thighs. Fifteen patients $(75 \%)$ expressed complete satisfaction. Five patients (25\%) reported return of some moisture to their hands and axillae. Conclusion: TUTS is minimally invasive, feasible and effective. It has a low complication rate. We recommend this procedure as the method of choice for the management of ULH.

Dr. Medhat M. Farghaly, FRCSI, FACS

Consultant General and Vascular Surgeon

PO Box 51601, Riqqa 53457 (Kuwait)

Tel. +965 9604501, Fax +9653919333

E-Mail medhat_f@hsc.kuniv.edu.kw 


\section{Introduction}

Primary upper limb hyperhidrosis (ULH) is excessive sweating beyond physiological needs, mainly in the palms, axilla and/or feet, without recognized etiology $[1,2]$. It is characterized by an overactivity of the sympathetic fibers passing through the upper thoracic sympathetic ganglia $\mathrm{T} 2-3$, resulting in excessive upper limb sweating [3]. It is a distressing and embarrassing condition affecting up to $1 \%$ of the population [4]. Most cases of ULH start in childhood and become aggravated during puberty [2]. This may cause severe psychological, social and occupational inconvenience.

The best treatment for this condition is upper thoracic sympathectomy at the T2-3 level, which was traditionally achieved by open surgical techniques. During the 1940s, a few pioneers started to excise upper sympathetic ganglia by thoracoscopy; however, the endoscopic approach, amply documented by Kux in 1954, did not gain widespread popularity until the 1980s [5-7]. Because of the simplicity of the procedure the indication for such a procedure has broadened to include patients who were considered previously unsuitable for the open approach [8]. It also includes cases of facial blushing and social phobia [9], vasospastic disease, angina pectoris and sympathetically mediated pain [10].

Three major contraindications to the procedure are considered: coagulopathy, poor lung function and pleural adhesions secondary to pulmonary tuberculosis, chronic bronchitis, previous thoracotomy or of uncertain origins. Pleural adhesions however are considered a relative contraindication, provided the surgeon knows anatomy and is well trained in performing the procedure [11].

We report our 2.5-year experience of the first thoracoscopic upper thoracic sympathectomies (TUTS) in Kuwait. This procedure was performed on 20 patients with ULH who presented to our surgical departments in the period from 16th March 1997 to 21st April 1999.

\section{Patients and Methods}

Only patients with severe hyperhidrosis were operated on. This was based on the severity of symptoms (damp + , wet ++ , dripping +++ ) and quality of life (annoyance + , debilitating ++ , social phobic +++ ).

Patients were examined clinically to assess the severity and location of hyperhidrosis. Basic hematological and biochemical investigations together with coagulation profile, thyroid function test and ECG were performed. Chest X-ray was performed to exclude any concomitant pleural thickening or lung pathology, which may be a contraindication to the procedure.

Patients were informed about the details of the procedure and the possible postoperative complications. A single dose of prophylactic antibiotic - ceftriaxone (Rocephine) $1 \mathrm{~g}$ - was given intravenously with induction of anesthesia.

The operation was performed bilaterally sequentially under general anesthesia in all patients, using one-lung ventilation with a double-lumen endotracheal tube. In 4 patients, a single-lumen endotracheal tube was used (the appropriate size was not available). On-table plain chest X-ray was performed to confirm its position. Patients were placed in the supine head-up position. The shoulders were slightly elevated, with both arms abducted to 70 degrees. The operation table was tilted 30 degrees towards the contralateral side.

Once the anesthetist had deflated the appropriate lung, a small incision was made over the 4th intercostal space in the anterior axillary line (to avoid injury to the long thoracic nerve). A pneumothorax was created by $500 \mathrm{ml}$ of carbon dioxide, driven through a Verres needle. The Verres needle was removed, and a $10-\mathrm{mm}$ trocar was inserted through which a 30-degree thoracoscope was introduced. Carbon dioxide insufflation was maintained at no more than $6 \mathrm{~mm} \mathrm{Hg}$ to avoid oxygen desaturation and cardiac arrhythmia. Two other ports $-10 \mathrm{~mm}$ and $5 \mathrm{~mm}$ in the $3 \mathrm{rd}$ and 4 th intercostal spaces, midaxillary line - were inserted in the first 28 cases. Later, we felt confident to perform the procedure using two ports only. In this approach, the sympathetic chain was easily identified by rolling it under the parietal pleura, running over the necks of the ribs in the upper costovertebral region (fig. 1). The stellate ganglion is usually not visualized as it is obscured by a 
Fig. 1. Thoracoscopic view of $\mathrm{T} 2-$ 4 of the right sympathetic chain. The azygos vein (AV) is shown medial to the sympathetic chain. The apex of the lung $(\mathrm{L})$ is retracted by an endo-clinch forceps. T1 is obscured by a pad of fat $(F)$.

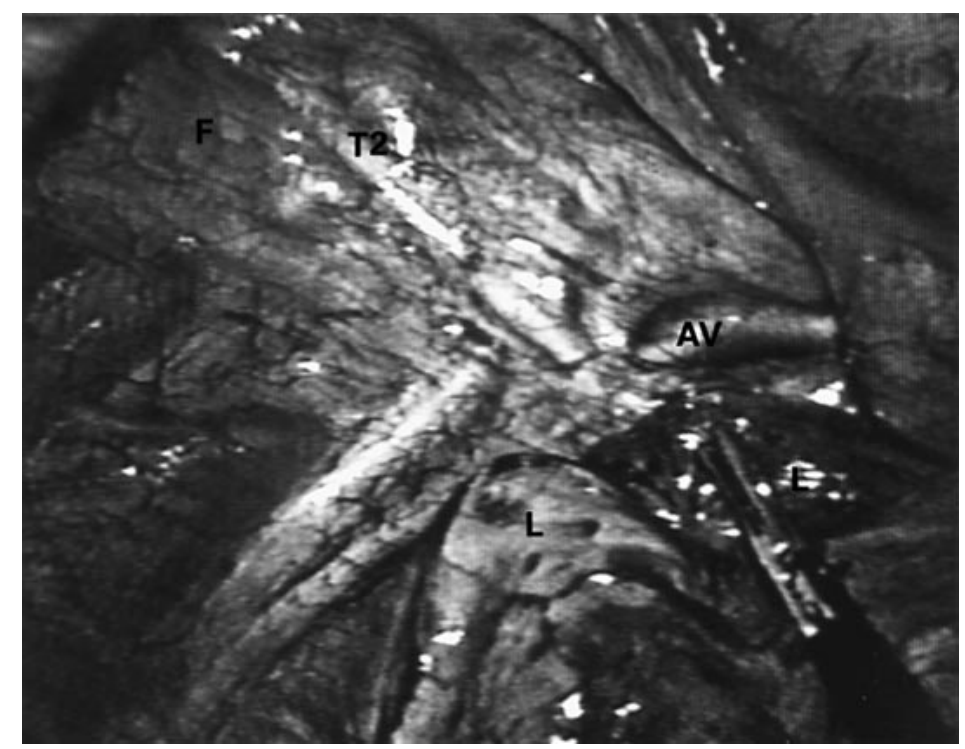

characteristic apical pad of fat and therefore protected from injury. The parietal pleura over the sympathetic chain is held by grasping forceps, and incised by an electrocoagulating hook. The sympathetic ganglia and intervening chain at the level of T2-4/5 were meticulously dissected, mobilized and excised from below upwards. Dissection included the postganglionic communicants and the accessory fibers of Kuntz whenever they were present. Clips were used, if indicated, to control bleeding. The Ultracision hook and scissors (Harmonic Scalpel, Ethicon Endodsurgery, Johnson \& Johnson GENII) were used in the last 8 procedures to replace electrocautery, as they cause no fulguration beyond $1 \mathrm{~mm}$. The removed ganglia were sent for histopathological confirmation.

At the end of the procedure, an Argyle $20 \mathrm{~F}$ intercostal chest tube was inserted in the lower midaxillary port, and connected to underwater seal. The anesthetist exerted continuous positive pressure for a few seconds to completely inflate the lung, which was usually expanded under endoscopic vision. The port sites were closed with coated vicryl 4/0 (polyglactin 910) subcuticular skin sutures. Chest tubes were removed 12$48 \mathrm{~h}$ postoperatively, after clinical and radiological confirmation of complete bilateral lung expansion.

Patients were closely observed during and after the operation for possible complications. The follow-up assessment of the late effects of sympathectomy on the

TUTS for Upper Limb Hyperhidrosis presenting symptoms was scored as completely better (highly satisfied), improved (satisfied) or unchanged (dissatisfied).

\section{Results}

Our study included 20 patients (18 males and 2 females) with ULH. Four of these patients $(20 \%)$ were Kuwaitis. Three patients $(15 \%)$ had positive family history of the same condition in 1 or more first-degree relatives. The mean age was 26.95 years (range 13-43 years).

All 20 patients had severe (grade +++) hyperhidrosis. All patients underwent simultaneous bilateral TUTS for hyperhidrosis, involving mainly the palms in 3 , palms and axillae in 2 and palms, axillae and feet in 15 . We had not encountered any case of isolated axillary hyperhidrosis. The T2-4 or T2-5 sympathetic ganglia were excised in 36 cases. In the rest, only the T2-3 ganglia were removed because of difficult localization. Two patients 
had bilateral dense pleural adhesions that necessitated sharp dissection. The operative time for the bilateral procedure ranged from 1 to $2 \mathrm{~h}$, average $1.42 \mathrm{~h}$. No conversion to open technique was necessary.

The postoperative follow-up period ranged from 5 to 30 months, average 16.3 months. No intraoperative or postoperative major complications were encountered. All had smooth recovery from anesthesia. One of the 2 patients who had dense pleural adhesions developed persistent right-sided pneumothorax. It resolved spontaneously with intercostal chest tube drainage, and the patient was discharged on the 7 th postoperative day. The rest were usually discharged on the 2 nd postoperative day. Two patients developed transient partial Horner's eye syndrome (ptosis and miosis) that resolved after 3 weeks in 1 and 8 weeks in the other. Four other patients developed nondistressing compensatory hyperhidrosis of the trunk and upper thighs. Three patients experienced postoperative pain in both arms and forearms, and 2 patients complained of back pain; all were transient and resolved spontaneously within 3-4 weeks.

Most patients (15 patients, 75\%) were highly satisfied with the outcome of the operation, having immediate and almost permanent improvement of their presenting symptoms. Five patients $(25 \%)$ were less satisfied and reported a return of some moisture to the hands and axillae 3-12 months after the operation. In no case was there a failure to achieve dry hands and axillae, or a return to the hyperhidrotic state. In fact, 11 of the 15 patients who had combined hyperhidrosis of their hands, axillae and feet (73.3\%) developed dry feet as well after TUTS.

\section{Discussion}

Sweating is a physiological response to heat, but excessive sweating confined to localized areas, such as the upper limb, may make work troublesome, and reduce quality of life. Therefore, the various surgical approaches are aimed at ablating the sympathetic supply to these areas. This can be achieved by excision of the ganglia (sympathectomy) [2,5], by interganglionic division (sympathicotomy) [6, $10,12]$, or by diathermy ablation of the sympathetic chain [13]. It can also be achieved by the new approach of clipping the interganglionic fibers, which is just as effective, and has the advantage of being reversible in cases of postoperative persistently distressing compensatory hyperhidrosis [14].

Our results confirm the previous report [6] that the 2nd-4th ganglia should be excised in case of upper limb hyperhidrosis, and at least the 5th ganglion should also be excised in cases of combined palmar-plantar hyperhidrosis. Drott [14] reported that in the case of palmar hyperhidrosis, a small majority preferred division over the 2 nd and $3 \mathrm{rd}$ rib in contrast to those who considered that the 2 nd alone was sufficient. In axillary hyperhidrosis, the participants were in agreement to include at least the $\mathrm{T} 3$, and a majority also the $\mathrm{T} 4$ ganglion. However, other authors suggested the inclusion of the 5th thoracic ganglion if axillary hyperhidrosis is the predominant symptom $[6,15]$.

In the past few years, TUTS has gained a wide acceptance as the method of choice for the troublesome ULH. The obvious advantages of the thoracoscopic approach to sympathectomy are the feasibility of performing bilateral simultaneous procedures, easy access to the 4th-5th ganglia, as well as minimal operative trauma, short stay in hospital, excellent cosmetic results, and a quick return to normal activities [2]. 
The immediate results of TUTS are gratifying for both isolated palmar and combined palmar and axillary hyperhidrosis. The findings of our study are consistent with the literature report on the efficacy of TUTS for upper limb hyperhidrosis, which approaches $100 \%$. In combined palmar, axillary and plantar hyperhidrosis, $73.3 \%$ of our patients reported decreased plantar sweating. This figure has reached up to $88 \%$ in other reports when the T2-7 ganglia were ablated [6].

The long-term results of sympathectomy are generally sustained success for hyperhidrosis, although less uniformly good for other conditions treated, especially Raynaud's syndrome [16]. At least $80 \%$ of patients remain dry. A further $15 \%$ have some return of symptoms, but still remain better when compared to the time of initial presentation $[15,17]$.

The most important complications of TUTS are persistent pneumothorax, compensatory hyperhidrosis, gustatory sweating, and Horner's syndrome. However, there are few rare complications which can be disabling or even life-threatening, such as long-lasting neuralgia of the ulnar nerve [18], thoracic duct and subclavian artery injury, and air embolism leading to intraoperative cerebral damage [7].

Postoperative chest pain is not a feature of TUTS because intercostal spaces admit 5and $10-\mathrm{mm}$ ports easily, thus causing less intractable chest pain. Other types of pain are transient, and disappear within 3-6 months [15]. Five of our patients experienced transient pain in either the arms, forearms or back.

Persistent postoperative pneumothorax may be encountered in cases of apical fibrosis and pleural adhesions. It usually results from damaging the visceral pleura during dissection [15]. Two patients in this study presented with unexpected pleural adhesions that required sharp dissection. One developed uni- lateral postoperative pneumothorax. Pleural adhesions, although considered a limiting factor using the single-port approach [19], are no longer a contraindication for TUTS. Coagulopathy and severe lung disease therefore remain the only contraindications [11].

Compensatory sweating is an exaggerated sweating in areas other than those denervated by sympathectomy and is the main reason for dissatisfaction in most series [12, 17]. It is a thermoregulatory response, probably based on central hypothalamic temperature regulation. Four of the patients $(20 \%)$ developed nondistressing compensatory sweating. It is reported in $25-75 \%$ of cases [18]; therefore patients should be informed of its occurrence at consent [20].

Horner's eye syndrome is usually temporary in TUTS. Its frequency varies between 0 and $9.3 \%$ [12]. There were 2 cases with such a complication. The stellate ganglion is not seen endoscopically as it is covered by a characteristic yellow pad of fat. However, variation in ocular nerve fiber distribution may occur, and the ciliospinal center may extend as low as the 5 th ganglion [15]. Therfore, cutting such fibers and thermal lesions from overcoagulation of the interganglionic fibers between the stellate ganglion and $\mathrm{T} 2$ are the most convincing explanations of unexpected Horner's eye syndrome $[2,12,18]$. The use of low-frequency diathermy or the Ultracision hook and scissors, which act by protein coagulation at the same site, may reduce its occurrence.

Finally, upper thoracic sympathectomy reduces myocardial oxygen consumption, and has an anti-ischemic effect. The parasympathetic response is predominant in such patients. Therefore, the vital signs must be closely monitored for any sudden vagal reflex during anesthesia in subsequent operations [22].

Med Principles Pract 2000;9:91-96 


\section{Conclusion}

TUTS is generally a safe procedure. It has the most gratifying results and lowest complication rate as compared to the conventional open methods. Our study contributes to its recommendation as the treatment of choice for ULH.

\section{Acknowledgment}

We acknowledge with thanks Dr. Abdulla A. AlAbassi, FRCSEd, for his great help in the preparation of this article.

\section{References}

1 Lery I, Ariche A, Sebaag G, Khodda $\mathrm{J}$ : Upper thoracic sympathectomy by thoracoscopic approach: A method of choice for the treatment of palmar hyperhidrosis. Ann Chir 1995; 49:858-862.

2 Cohen Z, Levi J, Pinsk I, Mares AJ: Thoracoscopic upper thoracic sympathectomy for primary palmar hyperhidrosis: The combined paediatric, adolescents and adult experience. Eur J Surg Suppl 1998;580: 5-8.

3 Noppen M, Herregadts P, D'Haens J, Vincken W: Cardiopulmonary exercise testing following bilateral thoracoscopic sympathectomy in patients with essential hyperhidrosis Thorax 1995;50:1097-1100.

4 Adar R, Kurchin A, Zweig A, Mozes $\mathrm{M}$ : Palmar hyperhidrosis and its surgical treatment: A report of 100 cases. Ann Surg 1977; 186:34-41.

5 Drott C: The history of cervicothoracic sympathectomy. Eur J Surg Suppl 1994;572:5-7.

6 Duarte JBV, Kux P: Improvements in video-endoscopic sympathicotomy for the treatment of palmar, axillary, facial and palmar-plantar hyperhidrosis. Eur J Surg 1998(suppl 580):9-11.

7 Cameron AEP: Complications of endoscopic sympathectomy. Eur J Surg 1998(suppl 580):33-35.
8 Weale FE: Endoscopic transthoracic sympathectomy; in Jamieson CW (ed): Current Operative Surgery: Vascular Surgery. London, Baillière, Tindall, 1985, pp 74-79.

9 Tetaranta T: Treatment of social phobia by endoscopic thoracic sympathectomy. Eur J Surg Suppl 1998; 580:27-32.

10 Rex OL, Dron C, Claes G, Gothberg G, Dalman P: The Boras experience of endoscopic thoracic sympathicotomy for palmar, axillary, facial hyperhidrosis and facial blushing. Eur J Surg Suppl 1998;580:23-26.

11 Lin CC, Mo LR: Experience in thoracoscopic sympathectomy for hyperhidrosis with concomitant pleural adhesion. Surg Laparosc Endosc 1996;6:258-261.

12 Lin CC, Mo LR, Lee LS, Ng SM, Hwang MH: Thoracoscopic T2sympathetic block by clipping - a better and reversible operation for treatment of hyperhidrosis palmaris: Experience with 326 cases. Eur J Surg Suppl 1998;580:13-16.

13 Weale FE: Upper thoracic sympathectomy by transthoracic electrocoagulation. Br J Surg 1980;67:7172.

14 Drott C: Highlights from the discussion. Eur J Surg Suppl 1998;580:4750.

15 Geraghty JG, Tanner WA: Endoscopic sympathectomy; in Macintyre IMC: Practical Laparoscopic Surgery for General Surgeons. London, Butterworth-Heinemann, 1994, pp 182-188.
16 Cambell WB, Cooper MJ, Sponsel WE, Baired RN, Peacock JH: Transaxillary sympathectomy: Is a onestage bilateral procedure safe? $\mathrm{Br} \mathrm{J}$ Surg 1982(suppl 69):S29-S31.

17 Zacherl J, Huber ER, Imhof M, Plas EG, Herbst F, Fugger R: Long-term results of 630 thoracoscopic sympathicotomies for primary hyperhidrosis: The Vienna experience. Eur J Surg Suppl 1998;580:43-46.

18 Ahn SS, Machleder HI, Concepcion B, Moore WS: Thoracoscopic cervicodorsal sympathectomy: Preliminary results. J Vasc Surg 1994;20: 511-519.

19 Kux M: Thoracoscopic endoscopic sympathectomy and axillary hyperhidrosis. Arch Surg 1978;113:264266.

20 Andrews BT, Rennie JA: Predicting changes in the distribution of sweating following thoracoscopic sympathectomy. Br J Surg 1997;84:17021704.

21 Lee LS, Lin CC, Ng SM, Au CF: The haemodynamic effect of thoracoscopic cardiac sympathectomy. Eur J Surg Suppl 1998;580:23-26.

22 Standfield NT: Thoracoscopic sympathectomy; in Bakerson-Brown S, Garden J (eds): Principles and Practice of Surgical Laparoscopy. London, Saunders, 1994, pp 604-608. 